\title{
One Step Forward in Cylinder Pressure Measurement
}

\author{
Miika Jussila Martin Giger Jürg Stadler Martin Kober
}

\begin{abstract}
Closed loop combustion control (CLCC) has established itself in a new generation of large engines such that the capability of using of different fuels, with very high engine efficiency, is now possible. The cylinder pressure sensors required for this application are subjected to high loads from pressure, temperature, and mechanical shock. A simulation led by sensor development approach is indispensable for achieving the best possible trade-off between measurement accuracy and service life. Patented designs such as diaphragm and ceramic feedthrough, paired with PiezoStar ${ }^{\circledR}$ Crystal technology, presented in this paper, form a reliable sensor unit that can satisfy and exceed the market requirements. Additionally, new approaches for transmission of electrical charge have been developed. This has enabled a significant increase in the robustness of signal transmission.

The trend towards higher mean effective pressures (MEP) on large engines means that the sensors must have a higher resilience to these pressure levels. In the testing and validation of service life, a specifically designed endurance test system has been developed for a validation of a service life greater than $10^{9}$ cycles. The endurance test system enables testing of sensors at an early stage.

Collaboration with engine manufacturers is essential for determining the environmental conditions, which define the requirements and boundary conditions for simulations and testing. Mounting location of the pressure sensor can affect the sensor service life due to environmental factors e.g. temperature and vibration. Collaboration with engine manufacturers allows for creation of robust installations and optimisation of interfaces. Field tests on appropriate target engines are still necessary as the final acceptance test, since the complete engine environment cannot always be fully replicated during the development stages.
\end{abstract}

\section{INTRODUCTION}

A core variable and performance metric in engine development is the cylinder pressure. Knowledge of this variable enables optimisations in the quantification of efficiency, emissions, and engine smoothness. Cylinder pressure measurement has thus become well established throughout the development of combustion engines, as it can optimize and shorten the overall development time. Increasingly stricter emission directives are being applied to large engines. It is not possible to achieve the required limits solely by combustion system optimisation for diesel engine applications. Gas and dual fuel engines are also unable to comply with the emission requirements without the use of additional hardware (e.g. exhaust gas after-treatment systems). However, a long-term cost benefit of gaseous over liquid fuels can be expected. Dual-fuel engines, which can be operated with the widest range of fuels, offer a high degree of investment security in the currently volatile energy market.

However, the increased complexity of the combustion process in dual-fuel operation, in combination with the requirement for maximum efficiency, demands additional control quantities for the combustion process. Cylinder pressure measurement offers high potential by providing engine cycle information at the source of the combustion, which can be used as a control parameter. Closed loop combustion control (CLCC) has established itself and proven its value for large engine applications. 
A new era has, therefore, dawned for cylinder pressure sensors in which long-term stability and robustness form an important additional requirement - without negatively impacting the signal quality. For this reason, Kistler has taken a new route in the development of next generation cylinder pressure sensors. In order to increase the robustness, new patented designs have been implemented and concepts have been realised for production that enable a better process control in order to meet the customers' quality demands.

The problem of reproducible loads is repeatedly faced in testing. Engines are certainly suitable for carrying out field tests, however, the specifications must be tested in detail already in the development phase, which is the reason why an endurance test system has been developed and installed by Kistler. This allows for an evaluation of sensor subassemblies, with regard to their ageing behaviour and service life. Consequently, it is now far easier to evaluate and optimise design concepts during development.

The environmental conditions that cylinder pressure sensors are subjected to, can be decisively influenced during the cylinder head design. Pressurisation, signal quality, temperature and vibration load on the cylinder pressure sensor are heavily dependent on the installation itself. These factors can be optimised during the design phase of the cylinder head. The selected installation can be evaluated via simulation and the robustness of the system can be improved by optimising the installation. A further design aspect is the life cycle maintenance. The selected seal type and mounting method, as well as the electrical interface, decide whether the system is able to perform the required task reliably and be easily exchanged by the service personnel.

\section{ENGINE REQUIREMENTS}

Numerous measurement principles for the measurement of pressure have been implemented and commercialized in specific applications. Capacitive, inductive, magnetoelastic, optical, resistance-based, and piezoelectric measurement principles are available. The major challenge for the measurement method used for cylinder pressure indication in large engines, is the combination of a large measuring range at high thermal and vibration loads, with the requirement for high-dynamic signal bandwidth and high measurement accuracy. For example, pressures up to $300 \mathrm{bar}$ with a resolution of less than $1^{\circ} \mathrm{crank}$ angle and non-linearity of $0.5 \%$ of the full-scale signal must be measured for a typical large engine application. Uncooled sensors can sometimes operate at temperatures of up to $350^{\circ} \mathrm{C}$. The cylinder pressure sensors can experience mechanical shocks of over $1000 \mathrm{~g}$, due to the impact of the intake and exhaust valves onto the valve seat rings. If the sensor is used in serial-production for closed loop combustion control, additional requirement is a long service life - one that has a greater than the usual "normally required" interval as a minimum. The impact of this requirement is that e. g. the sensitivity of the used sensor should not deviate significantly from its nominal value, even after a billion load cycles.

2.1. Selection of the sensor principle for cylinder pressure based engine control

In addition to the piezoelectric principle for cylinder pressure indication, primarily resistance-based measurements, which employ the piezoresistive effect, as well as optical methods, are sometimes applied.

For resistance-based sensors, the deformation of a current-carrying conductor, with a given electric resistance, changes the overall resistance value during this deformation. This occurs in part due to the geometric change of the conductor, but in particular due to the structural changes in the crystal lattice - a factor common to all semiconductor materials. When the measuring element is now applied onto the rear of the diaphragm of the pressure transducer (via brazing or sputtering techniques), or even exposed directly to the medium (direct chip exposure - DCE), a sensor response fast enough for cylinder pressure measurement can be achieved. However, it should be noted that the thermal load on the measuring element will then also increase, which can negatively impact the service life.

In fibre-optic sensors, the infrared light from an 
LED is emitted over an optical fibre onto the diaphragm of the sensor. The intensity of the reflected light is proportional to the pressure induced deflection of the diaphragm. The light intensity can be converted into an electrical signal in an opto-electronic circuit.

In piezoelectric sensors, force is transmitted by the diaphragm and applied to a piezoelectric crystal element. The force induces a mechanical deformation of the piezoelectric material, which produces a proportional electrical charge at the surface of the crystal. The electrical charge is converted into a voltage or current signal by an amplifier circuit.

Whilst the deformation of the diaphragm is primarily utilised for measurement in the two methods mentioned above, the piezoelectric sensor working principle is the transmission of force from the diaphragm onto the measuring element. The diaphragm is supported by the measuring element and can have relatively low thickness. The diaphragm itself, therefore, experiences only very small deformations during operation, which results in a fast response characteristic for the piezoelectric sensor, and which also contributes to achieving the required high number of load cycles.

Relatively high deformation of the measuring element (and thus the diaphragm) has to be implemented for piezoresistive thin-film sensors, because of their operating principle. Due to the lack of physical support in the case of a piezoresistive element, the diaphragm must have a comparatively high thickness. It should be noted that a thick diaphragm generally results in pronounced thermal shock behaviour, i.e. measuring error, which is caused by the rapidly changing thermal conditions in the combustion chamber during an operating cycle. Major errors are caused by thermal shock particularly during determination of energy conversion points and calculation of the indicated mean effective pressure (IMEP) based upon the measured cylinder pressure. Through data obtained on a single-cylinder engine test bench, during the comparison of various cylinder pressure sensors to a laboratory reference sensor, it can be seen that the tested piezoresistive sensors are only capable of fulfilling the criteria required by engine manufacturers, to a limited extent.

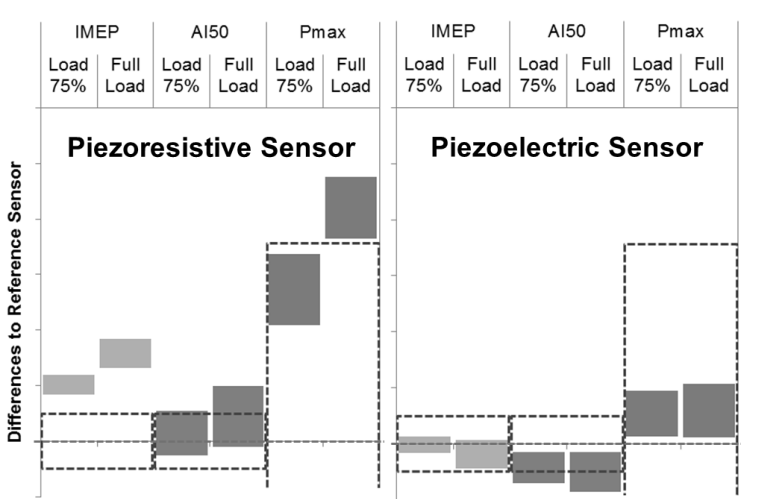

---Requirements for pressure-based engine control

Figure 1: Comparison of different on-board pressure sensor types to a reference pressure sensor on a single-cylinder engine test bench (scattering over at least eight specimens per type)

2.2. Integration of the pressure sensor in the cylinder head

Attention must be given to the installation position of the sensor to ensure that the cylinder pressure sensor can reach the required service life during the engine operation. Sensor installation close to the combustion chamber results in high cyclic heat flux on the sensor tip, but this installation method also enables proximity to the engine cooling water circuit, which provides a moderate temperature at the rear part of the sensor. Sensor installation far from the combustion chamber, behind an indicator cock, results in an even higher sensor temperature (unless further cooling measures are taken). In order to keep the sensor temperature as low as possible, the sensor tip (including the diaphragm) should be installed as recessed as possible, but so that the heat transfer from the sensor to the cylinder head and the cooling system is still sufficient. Recessed mounting results in a significantly lower cyclic heat flux and thus to a lower thermal shock. However, attention must be also given to the design of the sensor installation bore so that it does not cause signal distortions from acoustic gas oscillation. 


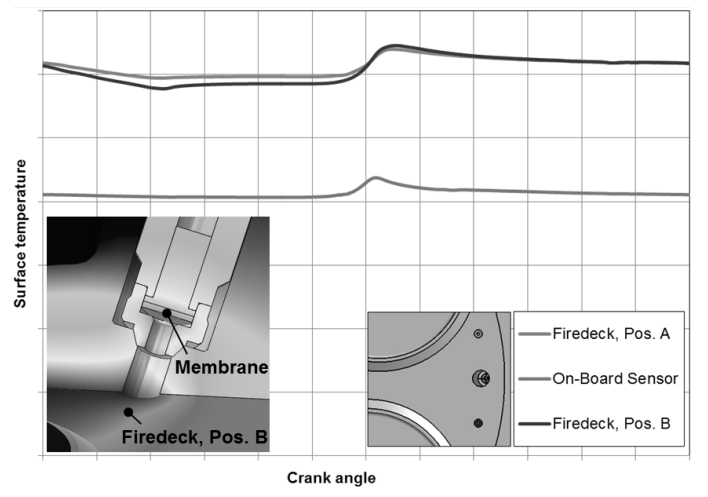

Figure 2: Surface temperature curves on the recessed sensor and firedeck close to the sensor from measurement and time-averaged component temperatures from simulation

Cylinder head deformation due to thermal expansion should not impact the measured pressure signal. This can be achieved in sensor design by extensive decoupling of the measuring components from the sensor casing. Vibrations from the impact of the valve head on the valve seat ring are transferred through the cylinder head to the sensor. Excitation of the sensor structure may result, which can mechanically damage sensor components e. g. friction locked electrical contacts. Optimisation of the sensor's location in the cylinder head can provide an appropriate solution.

Achievement of the required sensor service life not only calls for the optimisation of the sensor itself, but also of its integration in the engine. This development work can be carried out with the combined use of CFD and FEA calculations and the results can be verified on an engine test bench. This should ensure that the required benefits of the cylinder pressure based closed loop combustion control can be utilized over the entire service life of the sensor.

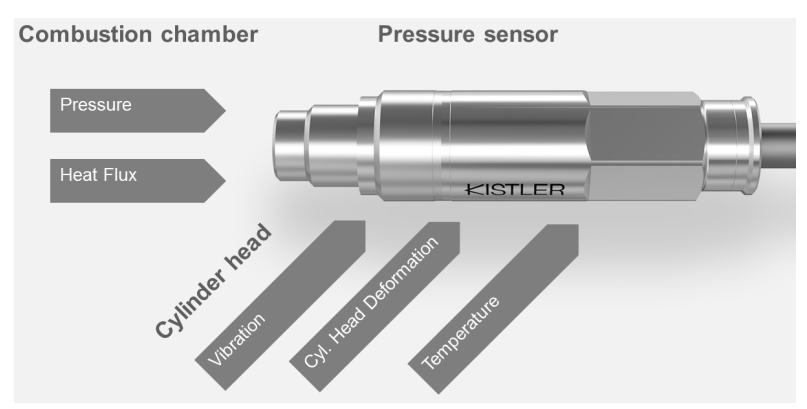

Figure 3: Pressure sensor with the corresponding environmental influences in the internal combustion engine

\section{SENSOR DESIGN}

The sensor type 6635A1 is a new development, for which specific design measures have been taken, with the goal of realising a robust, but also a very accurate sensor. Therefore, the sensor type 6635A1, has the following design features to meet the requirements for use in a large engine application:

$>$ Antistrain sleeve: Mechanical decoupling of the measuring element from the cyclic deformation of the cylinder head

$>$ PiezoStar $^{\circledR}$ Crystal: For minimum temperature dependence and disk-shaped geometry for maximum strength and stiffness

$>$ Ceramic feedthrough: Enables hermetic sealing of the measuring element and is part of the new metal to metal joint signal transmission

> Diaphragm: With new, patented geometry for high strength with a good measuring performance

> Signal transmission: The new signal transmission system is executed with metal to metal joints without moving parts to ensure reliable, wear-free signal transmission in harsh environments with high levels of vibration and mechanical shock

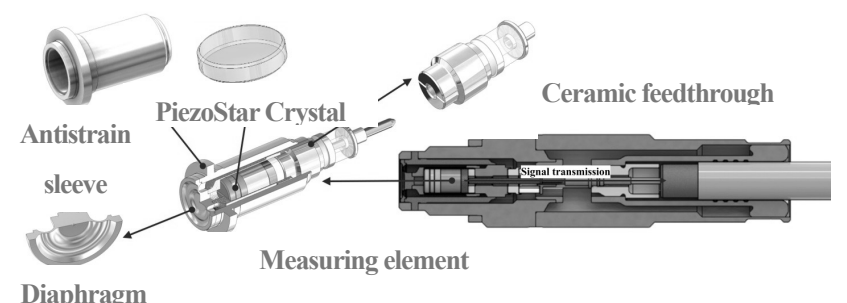

Diaphragm

Figure 4: Partial section through the measuring element with single parts and complete cylinder pressure sensor type 6635A 1 in half section

\subsection{Diaphragm}

When used in a combustion engine, the sensor 
diaphragm and the cylinder pressure sensor are subject to cyclic temperature fluctuation, caused by the flame front of the combustion. The cyclic temperature fluctuation results in a "cyclically occurring" measuring error, which is termed in combustion analysis as "thermal shock error" or also short time drift. Optimisation of a diaphragm for maximum accuracy, normally results in less strength for the diaphragm. This causes a trade-off between accuracy and strength. To counteract this conflict, a new patented design was developed. Through the additional flexibility in the curved diaphragm shape, the new design ensures a more homogeneous stress distribution with lower mechanical stress and very good measuring characteristics.

\subsection{Piezoelectric crystal}

The piezoelectric element is based on the advancement of the PiezoStar ${ }^{\circledR}$ Crystal technology developed by Kistler. The crystals have been grown and processed at Kistler since 1998. PiezoStar ${ }^{\circledR}$ Crystals belong to the calcium gallogermanate family - compounds similar to quartz such as langasite ( $\mathrm{La}_{3} \mathrm{Ga}_{5} \mathrm{SiO}_{14}$ ). PiezoStar ${ }^{\circledR}$ Crystals are characterized by the following properties:

\begin{tabular}{|l|ll|}
\hline & $\checkmark$ & High piezoelectric sensitivity \\
& $\checkmark$ & (up to 5x higher than quartz) \\
& $\checkmark$ & Minimum temperature dependence \\
& $\checkmark$ & High stability of the properties \\
$\checkmark$ & & Can be used at temperatures of \\
& $\checkmark$ & up to more than $800{ }^{\circ} \mathrm{C}$ \\
& $\checkmark$ & No phase transition up to \\
& $\checkmark$ & No twinning \\
$\checkmark$ & Growing process can be reproduced \\
& & on an industrial scale
\end{tabular}

\section{Tested and successfully used in high-quality piezoelectric sensors}

The latest crystal design from the PiezoStar ${ }^{\circledR}$ family has been optimised specifically for cylinder pressure sensors. It is characterized by extremely low sensitivity temperature dependence, high electrical insulation, and a high load capacity. The quality of the crystals is high due to the growing process, and they can thus ensure stable and reproducible properties over the entire service life of the sensor even at high temperatures. The piezoelectric crystal elements are machined internally at Kistler with the narrowest of tolerances, using the very latest 5 -axes CNC machines. The subsequent quality inspection, which is applied in the manufacturing process, ensures that only perfected crystal elements are installed in the sensor.

\subsection{Antistrain sleeve}

The antistrain design is based on a Kistler patent which has subsequently become established as the technical "state-of-the-art" design. This design has achieved decoupling of the piezoelectric sensor from the sensor casing. This means that the deformation (strain) occurring in the cylinder head is decoupled (as much as is physically possible) and the strain conditions in the sensor remain constant. This is required to attain a stable measurement signal with increasingly higher pressures, in combination with the consequential deformation of cylinder heads.

\subsection{Ceramic feedthrough}

Piezoelectric sensors require a very high electrical insulation resistance. The sensors are evacuated in order to avoid a decrease of the insulation resistance due to humidity in the sensor package, and to create an inert atmosphere. The ceramic feedthrough must ensure the hermetical sealing of the sensor package in all operating states over the service life. The 
assembly is exposed to an axial as well as a radial force in the sensor (see Figure 5). Primarily, this component provides the electrical insulation between the inner conductor and sensor casing, so that the electrical charge can be conducted to the cable. Furthermore, the ceramic feedthrough must ensure the hermetical sealing over a temperature range from -40 to $350{ }^{\circ} \mathrm{C}$.

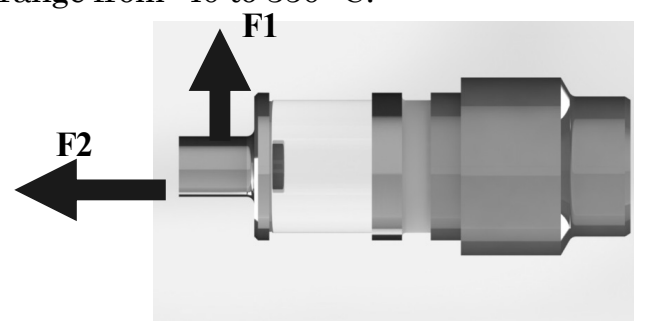

Figure 5: Ceramic feedthrough with load directions $\mathrm{F} 1$ and $\mathrm{F} 2$

\subsection{Reliable charge transfer}

Reliable signal transmission is essential in the low level signal range, such as the piezoelectric measurement technology, where currents in the picoampere range and voltages of a few millivolts have to be transferred. The signal path must be designed so that the electrical signal can be reliably transferred without any interruptions despite temperatures of $>200^{\circ} \mathrm{C}$ and high vibration loads during the entire sensor service life.

\subsubsection{Problems with existing signal transmission systems}

Sensors currently available on the market typically have at least one or multiple electrical connectors in the signal path. Electrical connectors have some advantages during the assembly process over other connection technologies; however, there are also some disadvantages. Use in the modern combustion engine with high acceleration loads from the closing of valves can result in occurrence of various closing and aging effects that can endanger the service life of the sensor.

\subsubsection{New signal transmission system}

The development of the new sensor had the objective of realizing as robust as possible electrical charge transfer. Therefore all moving parts in the charge signal path were eliminated. Typical products have several electrical connectors and crimped connections in the charge signal path between the sensor and amplifier. In the new design, all electrical connectors were eliminated and realized either as a single-piece or welded connection. Connection of the cable to the crimp sleeve is realised with a vibration proof crimped connection. The welded connections have the following advantages over electrical connectors and friction-locked systems:

1. No mechanical wear from vibration

2. No insulating layers on the contact surfaces from diffusion and oxidation at a high temperature

3. No moving components and thus no wear

Figure 6 shows the new patented signal transmission system. It consists of a single-piece electrode connected to the crimp sleeve by a welded connection. The crimp sleeve is connected to the inner conductor of the cable by a robust crimped connection. The new signal transmission system was validated by specific tests and also endurance tested with mechanical shock load. The test results clearly show that the charge transfer realized by a metal to metal joint is superior to the friction-locked electrical connections even at high temperatures. The new signal transmission system is, therefore, an important step towards reliable and robust signal transmission.

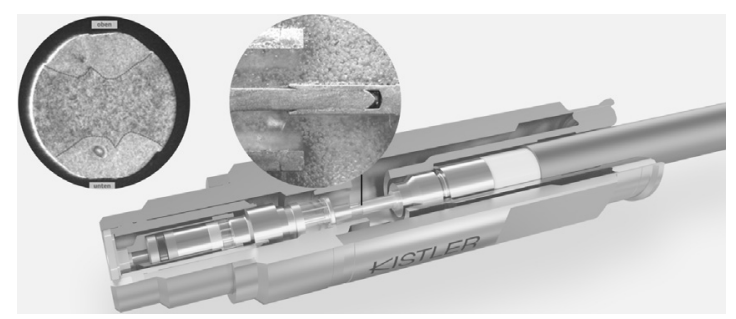

Figure 6: Sectional view of the sensor type $6635 \mathrm{~A} 1$ 


\section{SENSOR TESTING}

Testing the service life of pressure sensors for large engine applications is a considerable challenge, as the combustion engine, with its complex and harsh environment, is not easy to replicate and the requirement on the service life is very high. Therefore, the endurance test must be accelerated to obtain meaningful results during the development. During the development of the sensor type 6635A1, extensive endurance tests were carried out to determine and ensure the robustness and accuracy of the sensor over the service life.

\subsection{Service life test}

The endurance test was carried out on a hydraulic endurance test bench. Sinusoidal pressure loads, mechanical shock, and thermal loads can be combined and applied to the sensors being tested. The mechanical shock is generated with the aid of a piezo actuator and multiple sensors can be installed in a test adapter (see Figure 7).
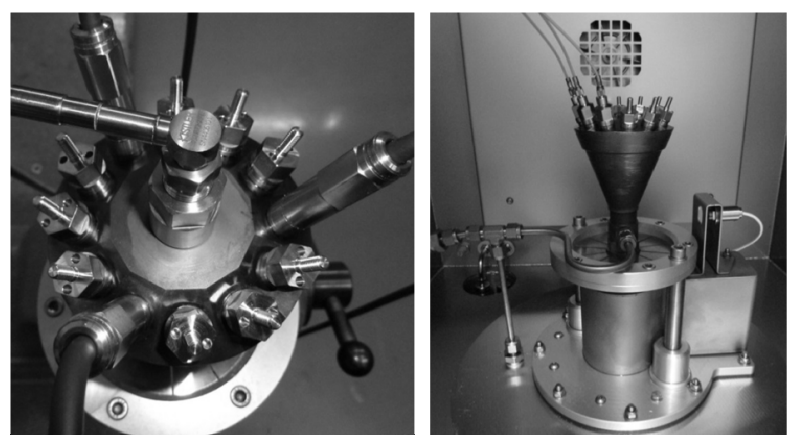

Figure 7: Left: Test setup with sensors under test; Right: Test adapter with sensors under test

Figure 8 shows an example of the combined shock and pressure load. When used in a 4-stroke engine, the sensor is loaded with mechanical shock caused by the intake and exhaust valves closing, as well as by the combustion. The shock generator is operated at twice the frequency of the pressure generator to simulate the engine environment. Additionally, the test adapter can be heated up to $350^{\circ} \mathrm{C}$. Test conditions similar to those in the engine, can thus be simulated with the endurance test bench.
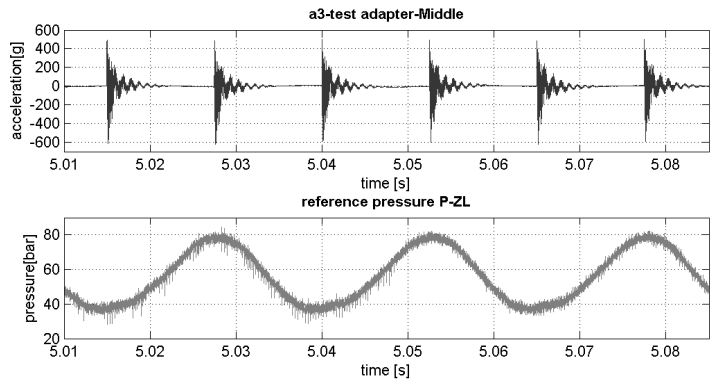

Figure 8: Example of acceleration and pressure signals during the endurance test

$10^{9}$ cycles with an operating pressure of 350 bar were tested in an endurance test during the development of the sensor. The endurance test was carried out with the following specifications.

Table1: Endurance test specification for the sensor type $6635 \mathrm{~A} 1$

\begin{tabular}{|l|l|}
\hline Test pressure & $350 \mathrm{bar}$ (sinusoidal) \\
\hline Shock (axial) & $\begin{array}{l}600-800 \mathrm{~g} \\
(\text { semi-sinusoidal })\end{array}$ \\
\hline Test adapter temperature & $250 \ldots . .350^{\circ} \mathrm{C}$ \\
\hline Number of cycles & $10^{9}$ \\
\hline Sensitivity change & $<1 \%$ FSO \\
\hline
\end{tabular}

One of the outstanding capabilities of the piezoelectric sensor is its long-term durability with constant measuring characteristics. An important feature for the stability of the sensor is the sensitivity change as a function of service life. Figure 9 shows that the sensitivity change over the entire testing period was $<0.4 \%$ FSO. Thus, proof could be provided that the long-term stability of the sensor is very good.

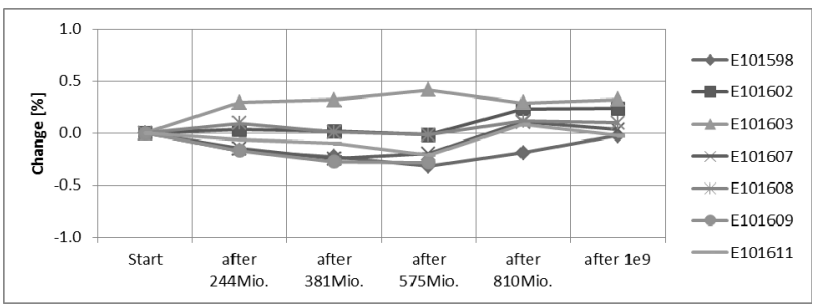

Figure 9: Sensitivity change as a function of the cycle number during the endurance test at 350 bar operating pressure. 


\subsection{Validation on engines}

The sensor manufacturer is able to carry out validation of the sensor on the newest combustion engines only to a limited extent, since the latest engines are usually not yet available in the field. Therefore, joint field and laboratory engine tests with selected customers are an effective method for determining the durability of the sensors. It must be ensured that the overload capacity is shown in specific tests, but on the other hand, field tests are used for validation of the stability of the sensor.

Determination of the measurement accuracy was tested on Kistler's own engines and also validated at universities with available test engines. With cyclic checking of the sensor properties on known engines, it was possible to determine and optimise the degradation behaviour.

\section{CONCLUSIONS}

Kistler has embarked in a new direction with the latest generation of cylinder pressure sensors for large engines. Consistent integration of simulation, process technology, and application know-how in the development processes is needed to ensure that the resulting product is able to meet the market requirements.

Installation in the cylinder head is an important aspect. The measuring quality, as well as the mounting reliability and service life of the sensor can be influenced by an early collaboration with the engine manufacturer. Explicit knowledge of the boundary conditions on the engine enables a better validation of simulations - this is consistent with current frontloading methods for state-of-the-art design processes. Engine manufacturers also have additional benefits from this working method due to the fact that the results can often give early identification of potential improvements on the engine.
High measurement accuracy and repeatability are essential to closed loop combustion control in today's engines. This basic requirement is fulfilled by the sensor type 6635A1, which is represented by the use of the latest PiezoStar ${ }^{\circledR}$ crystal technology paired with precision machined metal parts. To enable optimisation of the trade-off between robustness and measurement accuracy, a patented diaphragm structure has been developed pairing maximal strength with minimal thermal shock. The proven antistrain design has been used for additional decoupling of the sensor from the cylinder head, thus preventing crosstalk of cylinder head deformation.

The high mechanical shock-load on the sensors can result in limitations in signal transmission. A piezoelectric sensor delivers a low level of electrical charge. This is helpful in the fulfilment of ATEX regulations, but it places high requirements on the transfer of the charge signal. The elimination of all electrical connectors in the complete electrical charge path has reduced the risk of transmission errors to a minimum. This also includes the ceramic feedthrough, which has been designed to enable a failure free signal transmission over the service life of the sensor

The sensor was tested on different engines during which the stability and wear behaviour was determined over several thousand hours. Finally, a field test is always required on appropriate target engines to achieve a reliable implementation of cylinder pressure sensors in a closed loop control system. 


\section{References}

[1] A. Rempel, "Verbrennungsmerkmal geführte Regelung von mittelschnelllaufenden DF Motoren," in 9. Dessauer Gasmotoren-Konferenz, 2015.

[2] H. Kopecek, "Cylinder pressure based controls for robust operation of Gas Engines of high power density," in 9. Dessauer Gasmotoren-Konferenz, 2015.

[3] G. Gautschi, Piezoelectric Sensorics, Springer, ISBN 978-3-662-07600-8, 2002.

[4] R. Kuratle, Motorenmesstechnik, Vogel, 1995.

[5] A. Friedl, "Zylinderdrucksensoren für Großmotoren - Auswirkung der Sensoreigenschaften auf die Regelgüte bei Großmotoren,” in 12. Tagung „Der Arbeitsprozess des Verbrennungsmotors“, Graz, 2009.

[6] M. Giger and S. Brunner, "DRUCKSENSOR MIT EINER DRUCKRAUMSEITIG ANGEBRACHTEN MEMBRAN". Schweiz/Zürich Patent CH 2242015, 2582016.

[7] P. Engeler, "Antistrain". Patent EP230491.

[8] M. Giger and R. Buck, "PIEZOELEKTRISCHER DRUCKSENSOR UND VERFAHREN ZUR HERSTELLUNG DIESES PIEZOELEKTRISCHEN DRUCKSENSORS". Schweiz/Zürich Patent EP3124943, 07072016.

[9] M. Giger and R. Buck, "PIEZOELEKTRISCHER DRUCKSENSOR". Schweiz/Zürich Patent EP3124945, 07072016.

[10] M. Giger and R. Buck, "PIEZOELEKTRISCHER DRUCKSENSOR". Schweiz/Zürich Patent EP3124944, 07072016.
Dipl. Ing. Martin Kober

- LEC GmbH - Large Engines Competence Center, Graz University of Technology

\section{Authors}

Miika Jussila M.Sc.

- Kistler Instrumente AG

Martin Giger B.Sc.

- Kistler Instrumente AG

Dipl. Ing. Jürg Stadler

- Kistler Instrumente AG 